\title{
MATHEMATICAL SIMULATION OF THE TRANSFORMER DIFFERENTIAL PROTECTION, BASED ON THE TIME-IMPULSE PRINCIPLE
}

\author{
Mikhail V. Andreev ${ }^{1, *}$, Yuri S. Borovikov ${ }^{1}$, Nikolay Yu. Ruban ${ }^{1}$, and Aleksey V. Khlebov ${ }^{1}$ \\ ${ }^{1}$ Tomsk Polytechnic University, 634050, Tomsk, Russia
}

\begin{abstract}
The modern electric power systems (EPS) are large, multiparameter, stiff, non-linear and dynamic systems. Control and protection of such systems are extremely complex and urgent tasks. It is confirmed by the statistic of accidents in EPS: about $25 \%$ of severe accidents are caused by wrong actions of relay protection (RP) and automation of EPS. The 30 $\%$ of these incorrect actions are caused by inadequate setting. The goal of this work is to solve the problem of RPs setting through the usage of detailed models that take into account specific features of the RPs and processes in transducers. The paper focused on the solution of the problem for the transformer differential protection (TDP), based on the impulsetime principle - DZT-21/DZT-23. The presented research results confirm the effectiveness of the usage of the appropriate mathematical models for the problem solution. The presented fragments are a part of a single project aimed at the problem' solution for all the RPs devices.
\end{abstract}

\section{Introduction}

Due to unity and continuity of generation, distribution and consumption of electrical energy all equipment involved in the process: primary movers, generators, transformers, power transmission lines, electrical consumers and etc., including relay protection (RP) constantly interconnected in normal, emergency and post-emergency operational regimes.

Since the vast majority of this equipment is a dynamic elements, mostly non-linear and with very significant range of time constants $(>1000)$, any modern electric power system (EPS) [1] is a large, multi-parameter, stiff, non-linear, dynamic system. Research, design and utilization of such systems are very complex problems due to the difficulty of determining the influence of transients on the operation of RPs and the associated consequences for the operational regimes of the equipment and the EPS as a whole. According to the generalized statistics of accidents in the global electric power industry about $25 \%$ [2] of severe accidents are the result of incorrect actions of RP and emergency automation. In the $50-70 \%$ of cases wrong action of RP leads to the development of emergencies to severe system crash.

Because of development of the EPS simulation tools and, especially, creation in the Tomsk Polytechnic University of Hybrid Real-Time Power System Simulator (HRTSim)

* Corresponding author: andreevmv@tpu.ru 
$[3,4]$, which in this case is a source of primary information about the processes in the power system, became possible full investigation of the indicated problem, identification of its existence reasons and development on the basis of the results of these studies concept for solution of this problem.

\section{Mathematical simulation of relay protection}

The mathematical modelling is currently the only way to obtain information for the full spectrum of the processes and regimes of EPS. Depending on the specific tasks used models of the main and auxiliary equipment of power systems, including RP, varying details. In most projects, devoted to the evaluation of EPS stability, protection and automation reproduced as simplified models using the basic functions, such as a comparison with a threshold, timing and sending a command to circuit breakers. However, there are other approaches to the modelling of the RPs:

- Detailed modelling of physical processes in the RPs with the system of differentialalgebraic equations. This approach is mainly applied to study of electromechanical and electronic protection. The main drawback, according to the authors opinion, is the neglecting the measurement transducers (MT), which are mostly determine the nature of the identified processes.

- Detailed modelling of RPs operational algorithms. This approach is most popular at the moment, due to the fact that the researchers focused on the simulation of digital RPs (DRP) actively displacing other elemental bases. Here again, there are different approaches to the creation of such models. In some cases, models reproduce only a digital part of DRPs that implements the algorithm of its operation, in others - take into account a measurement and converting section, which includes primary and intermediate MT, as well as frequency filters in the form of dynamic models varying details. The complexity of development of the full models of specific DRPs caused by unavailability, in most cases, information about particular devices. Due to it a model designed on a base of data from manufacturer's guidelines.

Based on the analysis briefly presented above, it should be noted that the vast majority of projects for EPS modelling RPs models are not implemented properly, i.e. without taking into account of key software and hardware features of specific implementations and processes in the primary and intermediate MT. That is ultimately reduces the adequacy of the EPS model and simulation results.

Proposed by the authors and shown in a several publications, for example in $[5,6]$, approach aimed at creating universal all-regimes mathematical models of RPs, taking into account the mentioned features and processes. Such models can be used to solve the following scientific and practical problems: 1) investigation of the influence of the spectrum of transients in electric power systems on the operation of relay protection and identification of specific laws and theories; 2) reliable analysis and identification of the causes of incorrect actions of protection associated with the operation of the elements in a specific RP, development of recommendations for their improvement or modification; 3) testing and adequate setting of RP for the specific operational conditions in EPS.

This article presents fragments of the research results of the above problems on the example of transformer differential protection (TDP), based on the time-impulse principle DZT-21/DZT-23.

\section{Experimental results}


On the basis of the approach, which was indicated in the previous section, a universal allregimes mathematical model of DZT-21/DZT-23 is created. The phrase "all-regimes" means what a one model used for simulation the full spectrum of normal and abnormal regimes of EPS operation. Because of the limited volume of the article below shown a fragment of the model - second harmonic braking circuit (SHBC) of DZT-21/DZT-23: equivalent circuit (Fig. 1a), directed graph (Fig. 1b) on the basis of the equivalent circuit, transfer function (1) and the differential equation (2).

The transfer function:

$$
W_{\text {secHarm }}(p)=U_{\text {secHarm }}(p) / U_{\text {Filter }}(p)=\left(K_{1} \mathrm{p}+K_{2}\right) /\left(K_{3} \mathrm{p}+K_{4}\right)
$$

where $K_{1}, K_{2}, K_{3}, K_{4}$ - coefficients determined by RLC-parameters of SHBC.

The differential equation:

$$
d\left(K_{3} u_{\text {secHarm }}(t)-K_{1} u_{\text {Filter }}(t)\right) / d t+K_{4} u_{\text {secHarm }}(t)-K_{2} u_{\text {Filter }}(t)=0
$$

Implementation of the synthesized mathematical model of DZT done through transforming the model presented in the form of a system of differential equations into a program code, integrated into the software of HRTSim. Another option also was done autonomous software with capability of downloading information about the processes in the EPS from simulators. The main advantage of the first option - it is an opportunity of real-time tracing the response of EPS on the action of RPs and analyzing consequences.

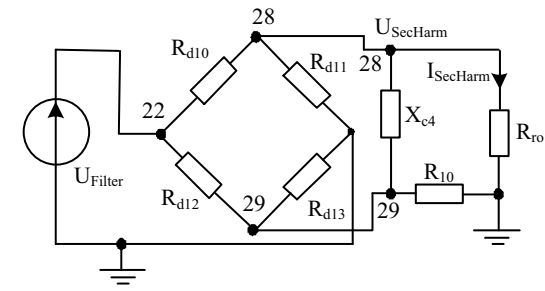

a)

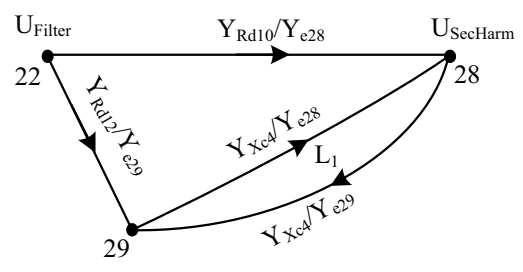

b)

Fig. 1. Equivalent circuit (a) and graph (b) for second harmonic braking circuit: UTAV2 transreactor output voltage; $Z_{\text {load }}$ - load impedance; $U_{\text {Filter }}$, $I_{\text {Filter }}$ - second harmonic filter output voltage and current; $\mathrm{X}_{\mathrm{L}}$ - inductive resistance.

Currently two basic approaches to validation of RPs mathematical models are applied: 1) comparison of results of operation of mathematical models and real devices; 2) comparison of characteristics of particular elements models with theoretical information available in open sources. The first approach is difficult to implement due to difficulty of information extraction from particular nodes of the real device, as well as the lack of access to the equipment. For validation of the DZT-21/DZT-23 model the second approach was applied.

The spectrum of regimes for model testing includes all possible internal and external short-circuit (SC), as well as inrush current regime. Below presented are fragments (Fig. 2 and 3) of the research of the DZT-21/DZT-23 mathematical model in regime of external three-phase SC on the $220 \mathrm{kV}$ bus-bar - the highest voltage (HV) of protected autotransformer.

According to the oscillograms (Fig. 3b) a incorrect tripping of BC complect of protection is occurred. This fact show a ouput signal of tripping-time-delay element of operating element. 


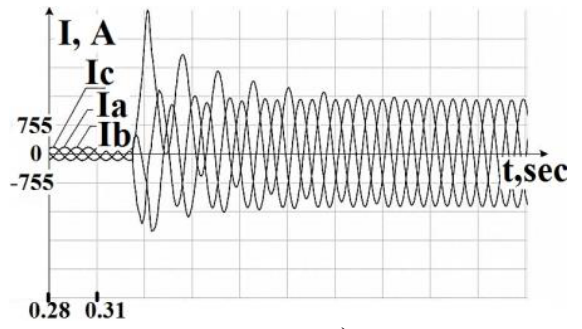

a)

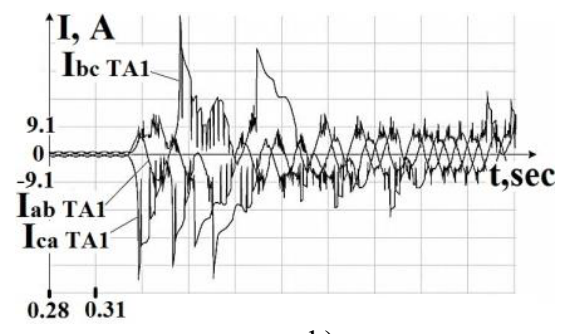

b)

Fig. 2. a) Oscillograms of phase primary currents of the HV side of the autotransformer; b) oscillograms of linear current at the output of current transformers on the HV side.

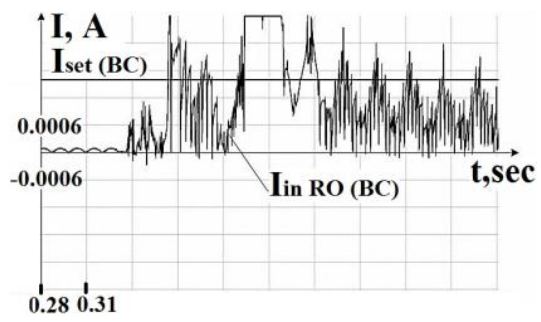

a)

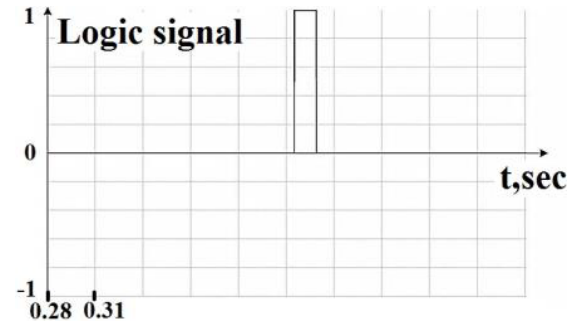

b)

Fig. 3. a) Oscillograms of input currents of operating element of BC complect; b) oscillograms of output signal of tripping-time-delay element of BC complect.

\section{Conclusions}

The done studies allow analysing the errors of specific elements of the TDP, testing the setting and correct them based on the analysis of RP operation in different regimes. The research results allow confirming the fact of a significant impact of transients in EPS on the TDP operation and make some conclusions about the effectiveness of time-impulse principle and effectiveness of the protection work as a whole. The presented results of studies in combination with others, obtaining of which is planned in the near future, will form the basis of the concept for solution of the problem indicated at the beginning.

The reported study was funded by RFBR, according to the research project No. 16-3860043 mol_a_dk.

\section{References}

1. M. Mazurov, A. Nikolaev, N. Lozinova, IEEE Russia Power Tech, 1 (2005)

2. A. Kuzmichev, E. Konovalova, S. Sakhorov, A. Zakharenkov, Relay protection and automatization, 1, 60 (2012)

3. A. Gusev, A. Suvorov, A. Sulaymanov, IOP Conf. Ser.: Mater. Sci. Eng., 93, 1 (2015)

4. R. Ufa, V. Sulaymanova, Yu. Borovikov, MATEC Web of Conf., 37, 01061 (2015)

5. Yu. Borovikov, A. Sulaymanov, M. Andreev, IOP Conf. Ser.: Mater. Sci. Eng., 93, 1 (2015)

6. A. Suvorov, Y. Borovikov, A. Gusev, A. Sulaymanov, M. Andreev, N. Ruban, R. Ufa, Electrical Engineering (to be published) 